Vol.15, No. 55, April, 2020, 663-671

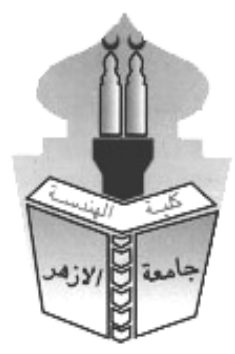

\title{
ACHIEVING THE QUALITY OF THE LIGHT ENVIRONMENT INSIDE HOTEL ROOMS USING SMART SYSTEMS
}

\author{
Dalia Magdy Mohammed Morci Kassem*, Ahmed R. Abdin, Mohsen Abo-Elnaga \\ and Gehan A. Elsaid
}

Architecture Department, Faculty of Engineering, Cairo, University Egypt

*Corresponding Author E-mail: arch.daliamagdy@yahoo.com

\begin{abstract}
This research presents how to achieve the quality of the photovoltaic environment for hotel accommodation in hotels using smart systems, as reducing energy consumption rates is a major goal of the smart hotel design, which is directly related to the efficiency of the internal environment of the guest rooms, the higher the quality of the photovoltaic environment, the lower the rates Energy consumption, as it achieves a quality environment in the hotel's accommodation rooms, achieving visual comfort.

A high-quality indoor environment is provided with attention to the quality of the "thermal environment" and the quality of "indoor air environment" as well as a good "light environment", which affects the visitor's sense of comfort inside the room through the elements of perceptual components of that internal environment, as the quality of the internal environment is linked A direct correlation to achieving energy efficiency, and this research will explain how to achieve the quality of the photovoltaic environment in smart hotel rooms, thus achieving comfort for visitors and reduces energy consumption by using smart systems.
\end{abstract}

\section{KEYWORDS: Quality Of The Light Environment, Guest Rooms, Smart Systems, Day Light, Artificial Light}

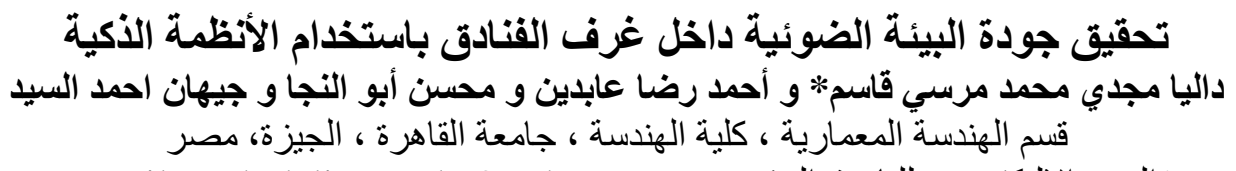

*-mail: arch.daliamagdy@ yahoo.com البريد الاليكتروني للباحث الرئيسية

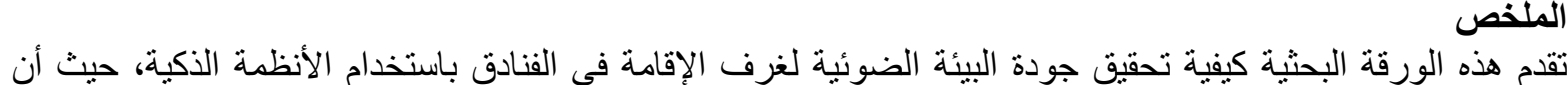

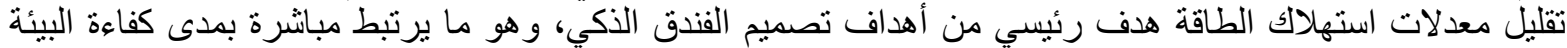

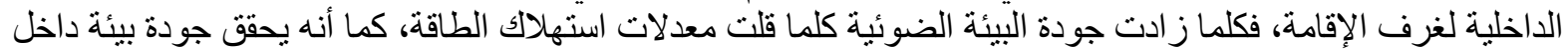

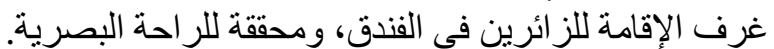

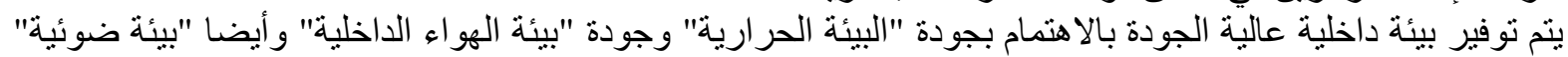

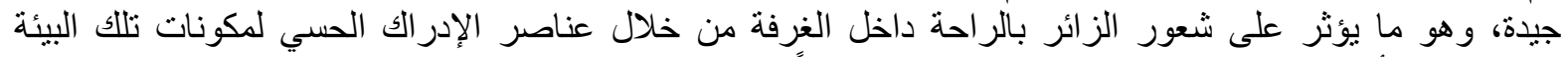

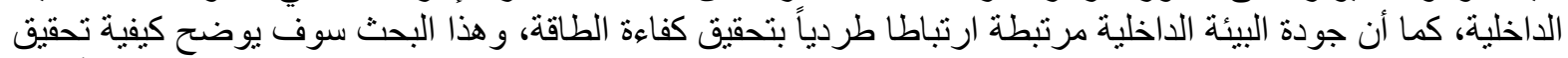

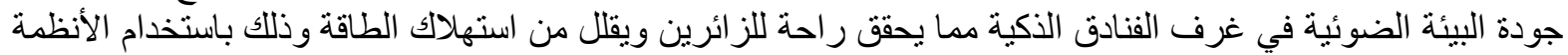
الذكية.

الكلمات المفتاحية: جودة البيئة الضوئية ، غرف الإقامة، أنظمة ذكية، إضاعة طبيعية ، إضاءة اصطناعية. 


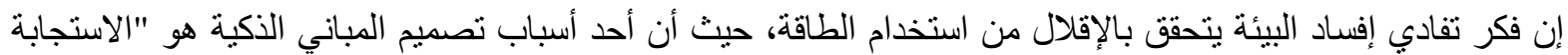

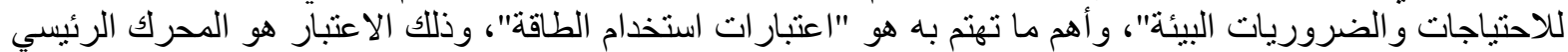

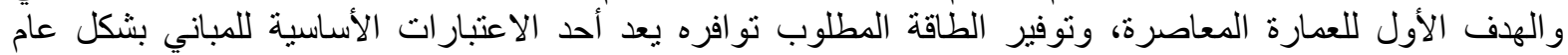

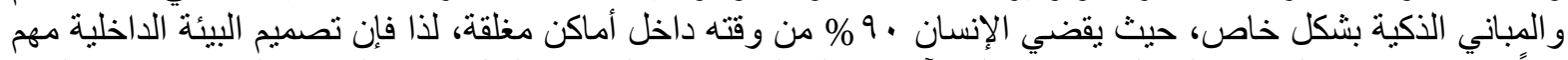

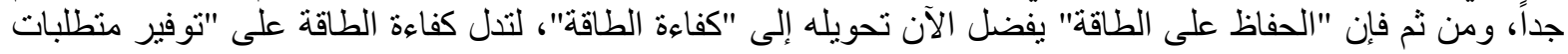

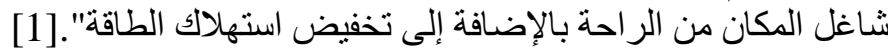

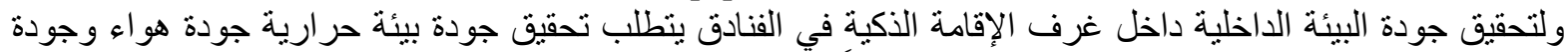
بيئة ضوئية وصوتية، وما يعود هذا على تحقيق أيضاً كفاءة الطاقة، ولتحقيق استخدام الأنظمة الذكية لتحقيق الهيقة الهدفين

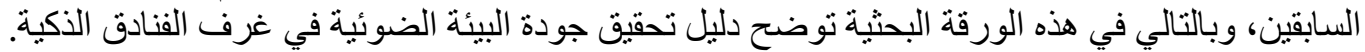

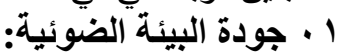

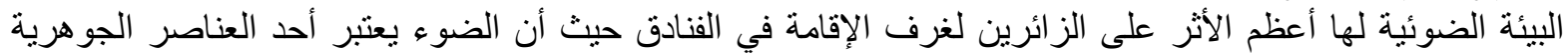

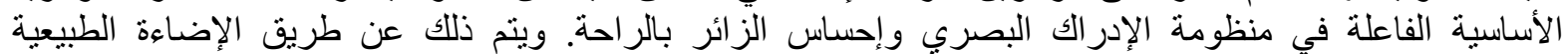
و والصناعية. ولما للإضاءة الطبيعية من مميزات لإية يمكن الإضاءة الصناعية تعويضها وهى تحقيق الراحة البصرية و النفسية ونوفير

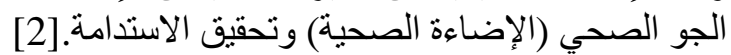

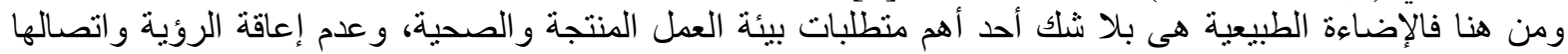

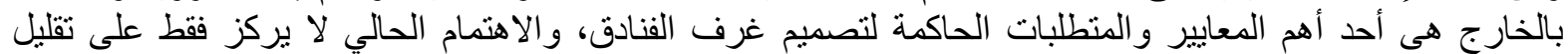

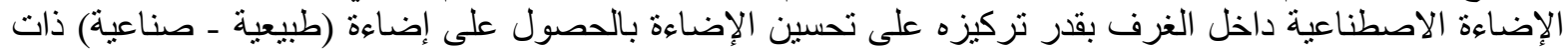

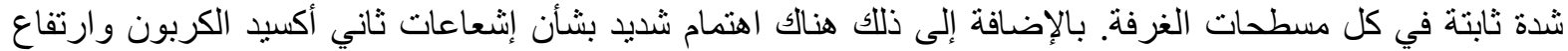

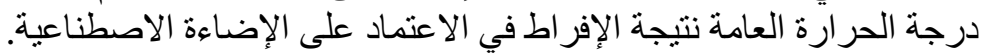
و عليه فالهدف من نظام الإضاءة الطبيعية في الواجهات الذكية هو التفاعل مع نظام الإضاءة الإهاءة الاصطناعية والتكامل معه لتحقيق هدفين أساسين: أـ خفض معدلات استهلاك المبنى من الطاقة الكهربائية المستهلكة في الإضاءة الاصطناعية.

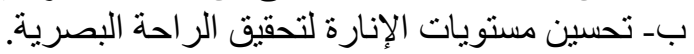

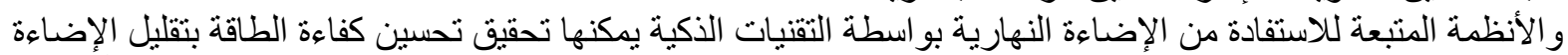

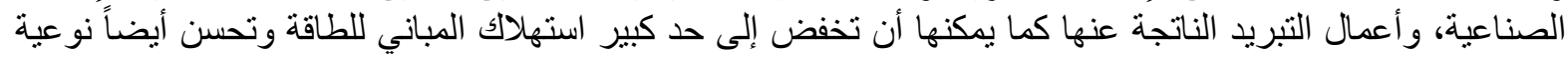
الإضـاءة في البيئة الداخلية بشكل ملحوظ. [3: و على ذلك فإن هنالك نو عان من "وسائل التحكم في الإضـاءة الصناعية المستجيية للإضاءة الطبيعة" يلتزم تو افرها لتحقيق

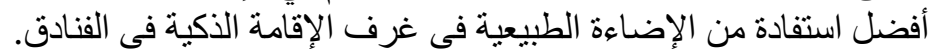

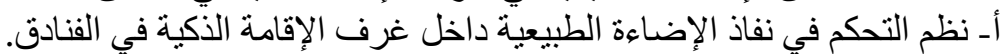

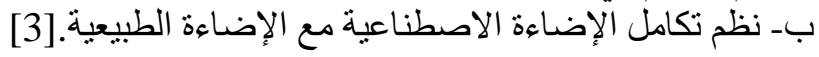

r · ن نظم التحكم في نفاذ الإضاءة الطبيعية داخل غرف الإقامة الذكية في الفنادق:

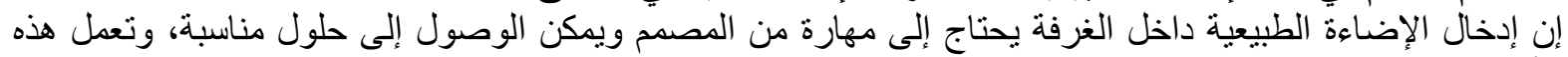

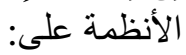

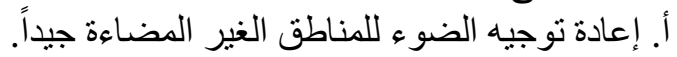

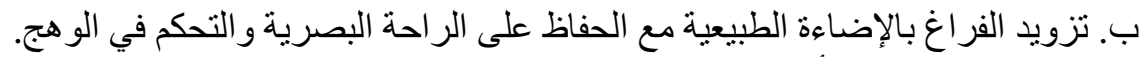

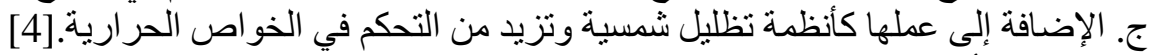

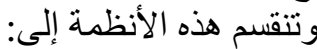

( ) - أنظمة الإضاءة الطبيعية المزودة بأنظمة التظليل:

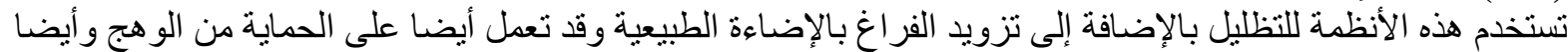

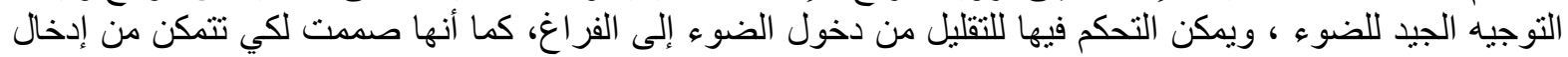

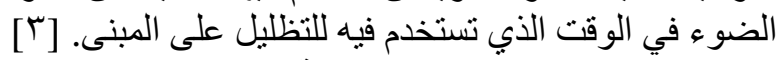

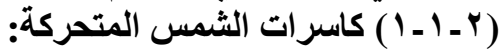

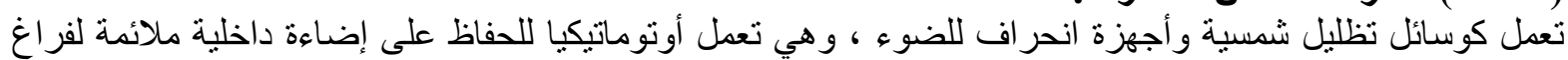

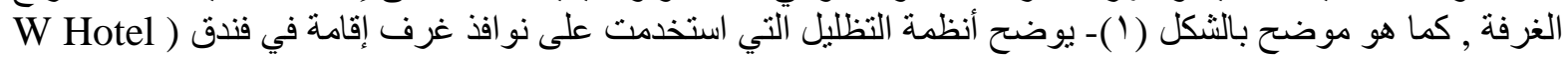
(Dubai 

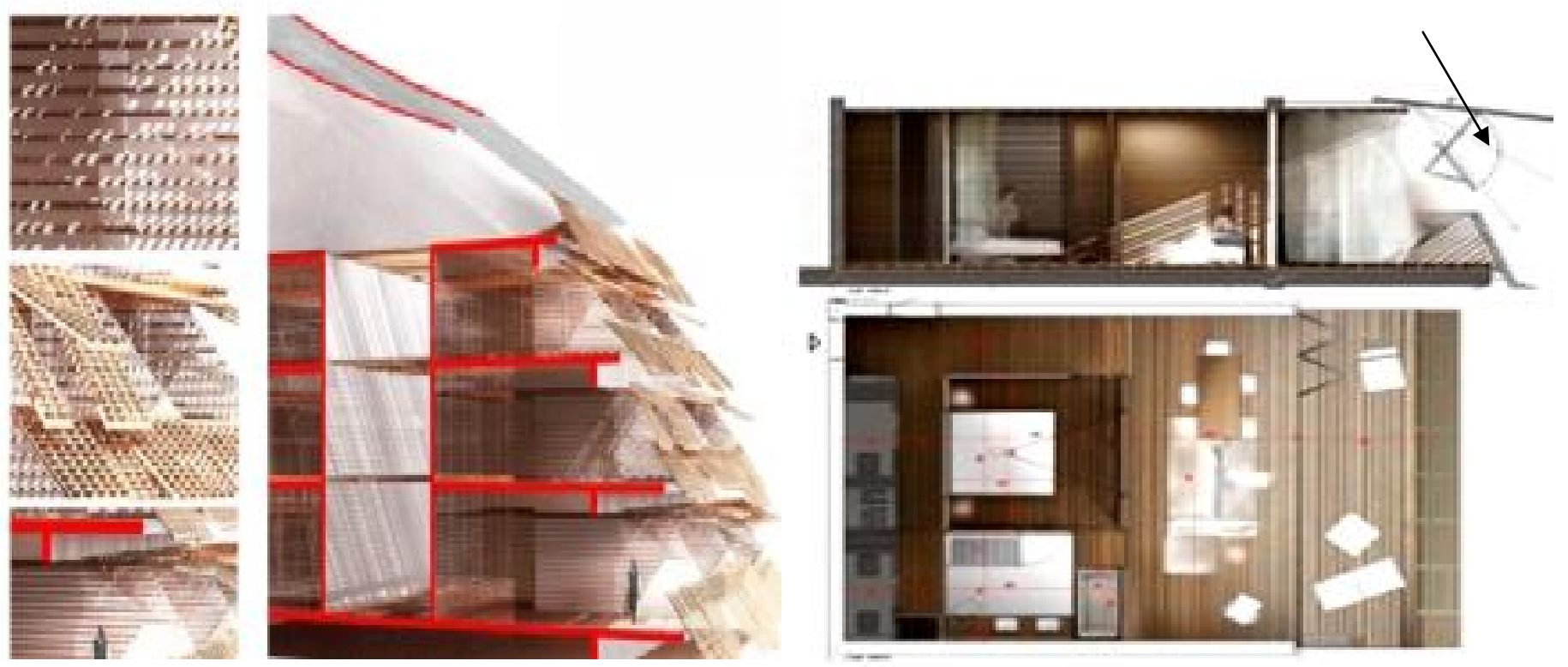

كاسرات شمسية

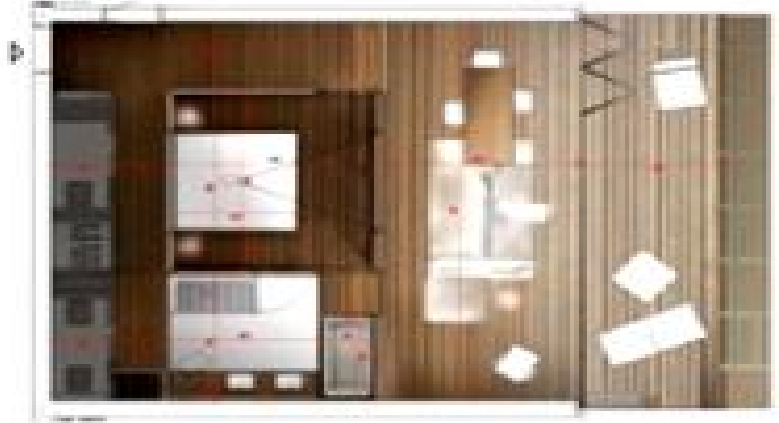

\section{قطاع يوضح احد انظمة التظليل في غرف الفندق}

شكل ( ) - يوضح أنظمة التظليل (الكاسرات الثمسية) في فندق (WHotel Dubai) - دبي

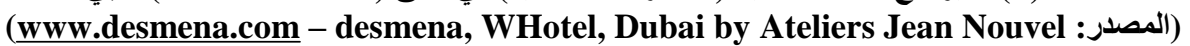

:(Holographic Optical Element) HOE

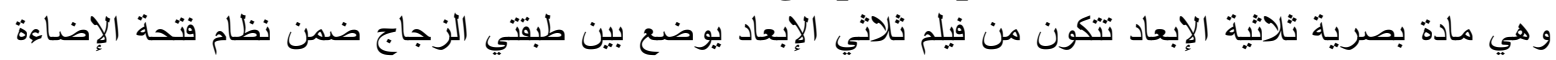

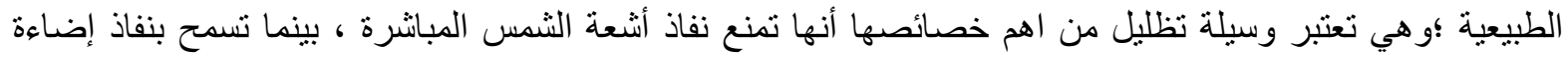

السماء الغير مباشرة [4]كما هو موضح بالثكل (r).

\section{مسقط افقي للغرفة}

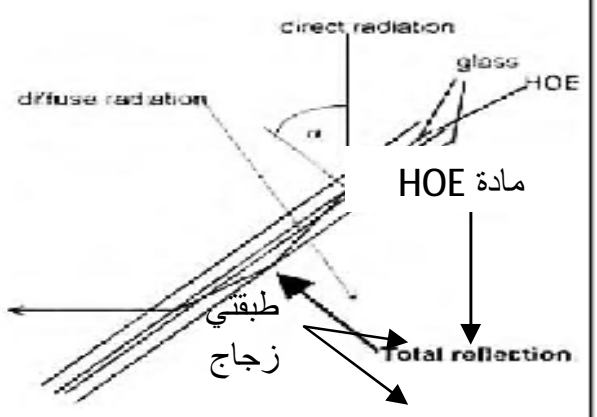

شكل (r) - يوضح المبدأ الأساسي لوظيفة (HOE) بين طبقي الزجاج

(المصدر: Richard Watkins, Daylight in Building, 2010, computer Science)

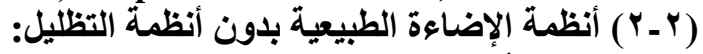

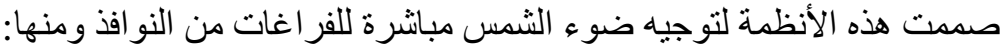

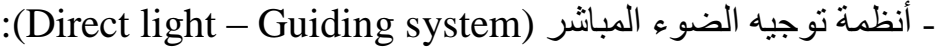

نظام (Laser cut panel) : و هي عبارة عن وحدات رفيعة مصنو عة من الاكليريك الثفاف مقسمة بو اسطة (laser cut)

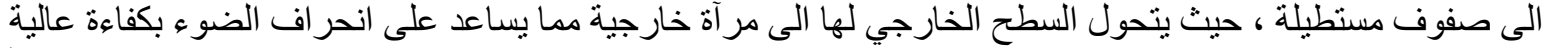

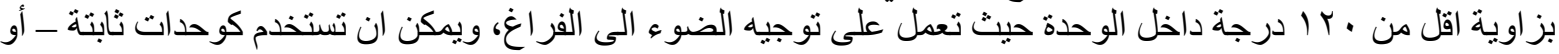

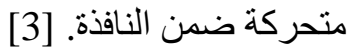


هى تعمل على توجيه ضوء الثمس المبانثر إلى الفراغ بدون أي تأثثرات جانبية للوهج أو إحساس بالحرارة كما هو

موضح بالثكل (r). (r).
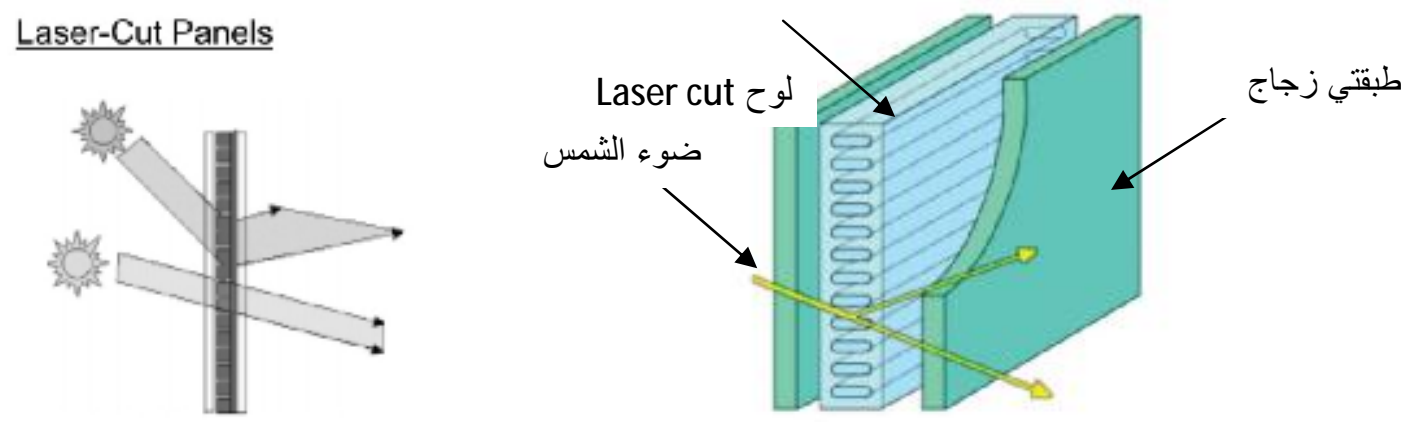

Laser cut panel شكل (") - نظام

(المصدر: Richard Watkins, Daylight in Building, 2010, computer Science)

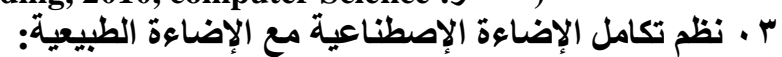

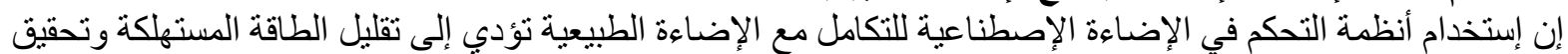
جودة البيئة الضوئية المطلوبة لغرف الإضف الإقامة الذكية في الفنادق.

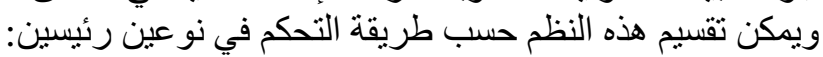

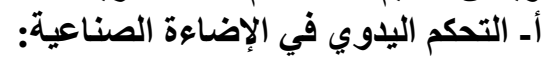

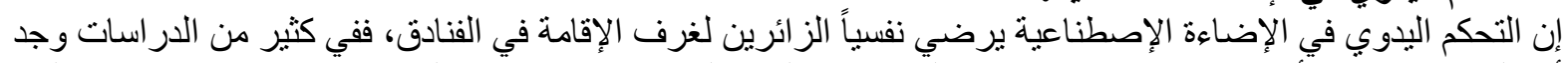

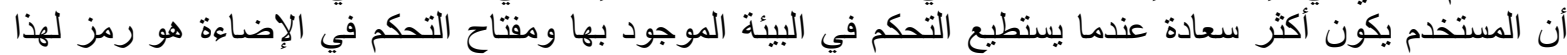

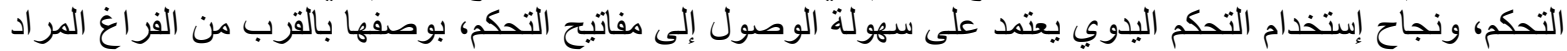
إضاءته مثل فر اغ النوم بجانب السرير، منطقة الجلوس. كما موضح بالثكل (ع ).
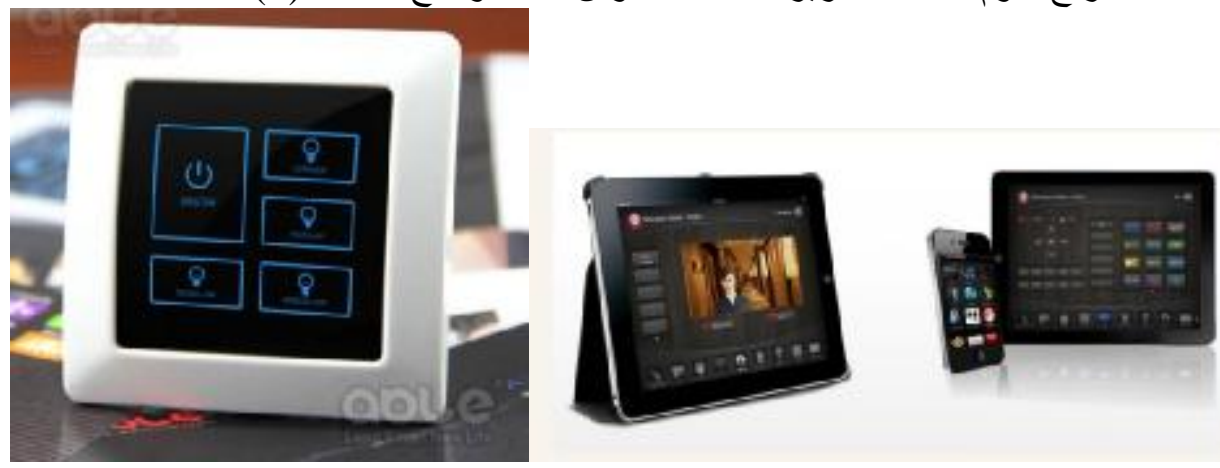

شانشات الكترونية تعمل مع النظام الذكي(Smart system)

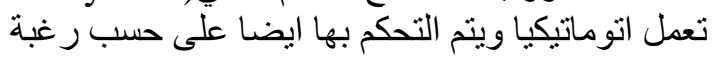

شكل ( \&) - طرق التحم اليدوي الز ائرين في الغرفة.

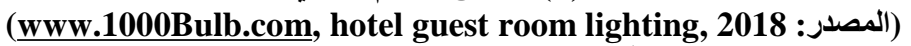
بـ التحكم الأوتوماتيكي في الإضاءة الصناعية:

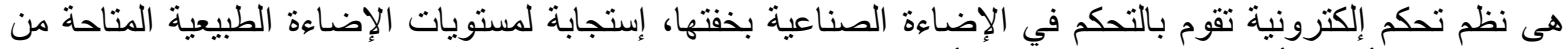

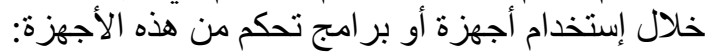

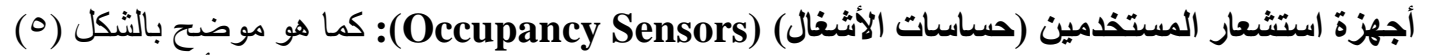

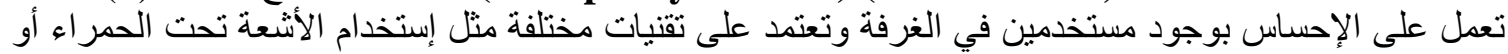
إستخدام الموجات فوق الصوتية أو الموجات الدقيقة (Microwaves). 

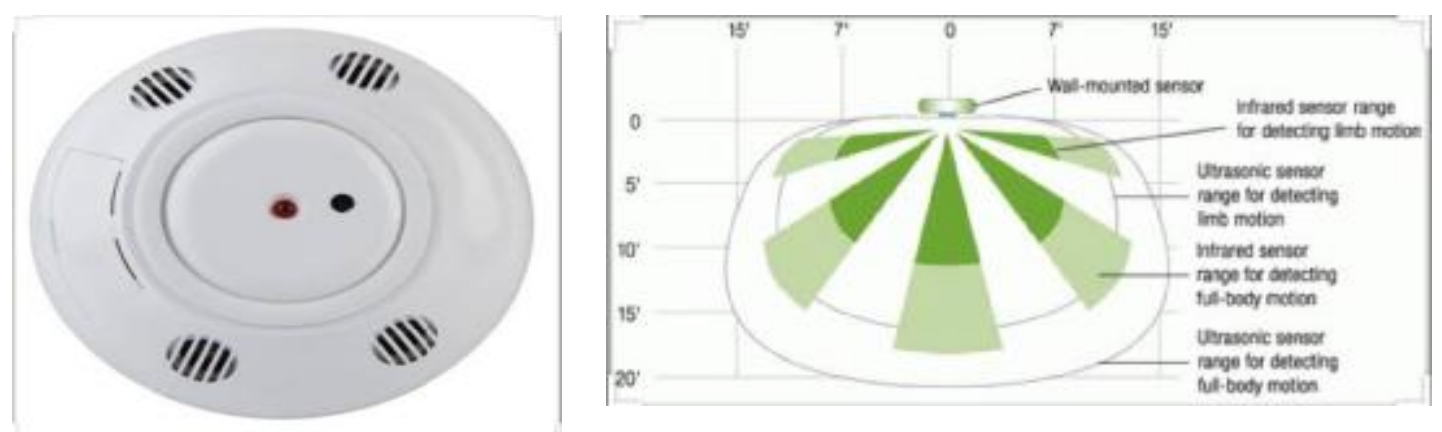

شكل (•) يوضح شكل لأحـ الـ(Occupancy Sensors) المستخدمة في الـSmart guest rooms (المصدر: (www.bowinlock.com, 2017 )

(الحساسات الضوئية (Photo Sensors):

تعتمد الحساسات الضوائية ببساطة على تحديد مستوى الإضـاءة الطبيعية المنوفرة ومن ثم العمل آلياً على تحديد

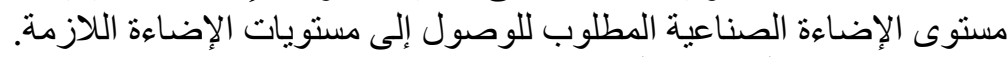

؛ · نظم الإضاءة الإكية المتكاملة (Intelligent Integrated Lighting):

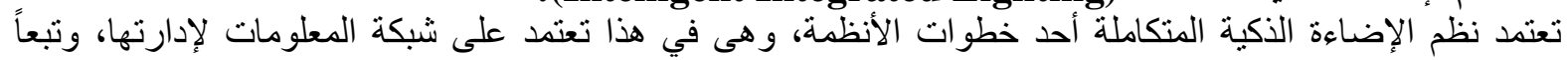

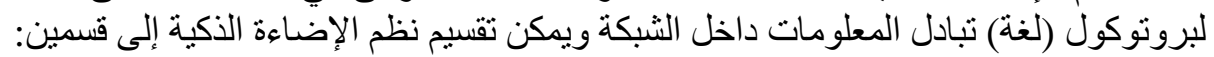

جدول (1) يوضح الفرق بين البروتوكولات المغلقة والمفتوحة وأفضلية اختيار النظام المفتوح (المصدر: عمل البران الباحث).

\begin{tabular}{|c|c|}
\hline $\begin{array}{c}\text { نظم تعتمد على البروتوكولات المفتوحة } \\
\text { (Open Protocol) }\end{array}$ & $\begin{array}{c}\text { نظم تعتمد على البروتوكولات المغلقة } \\
\text { (Closed Protocol) }\end{array}$ \\
\hline 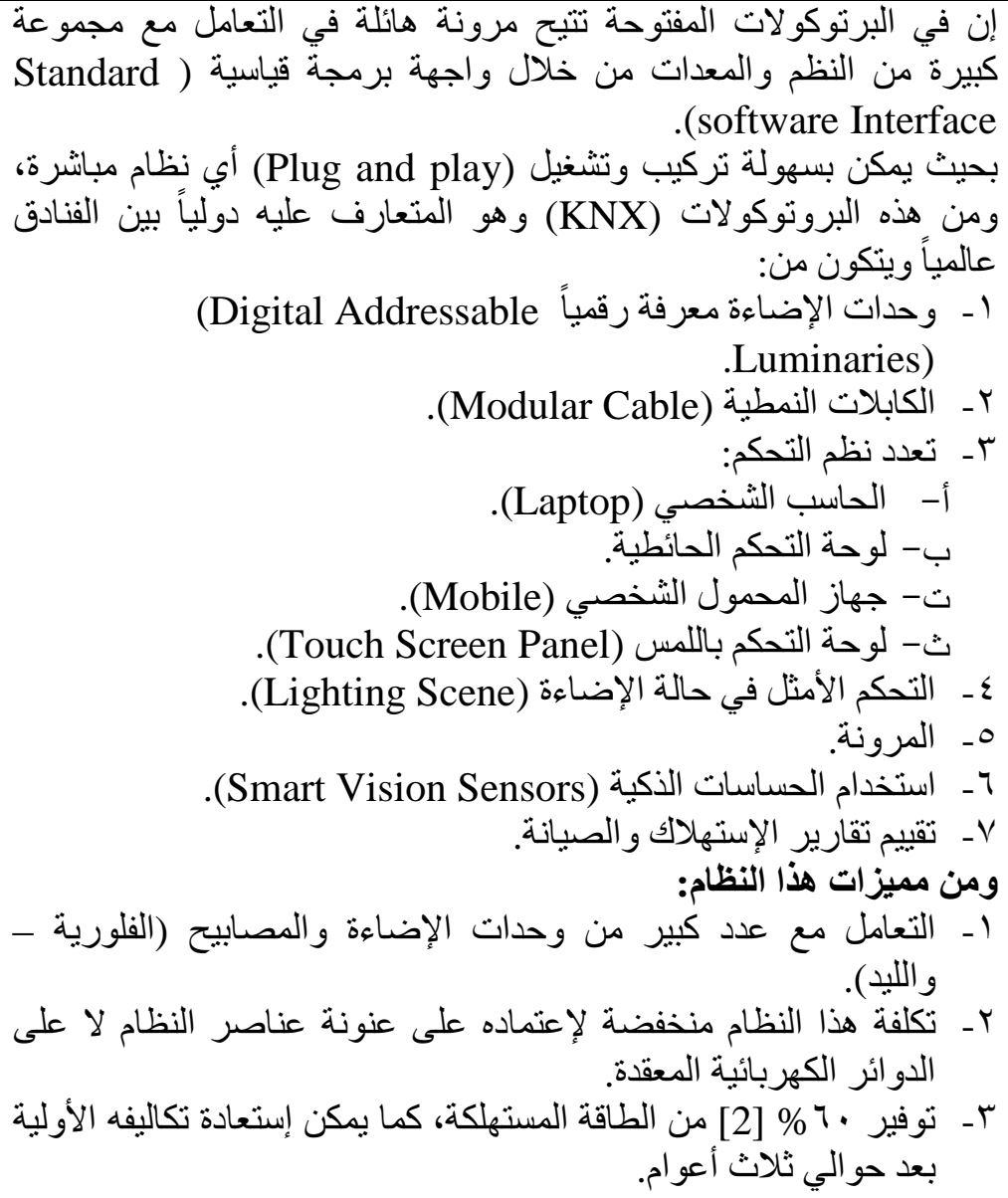 & 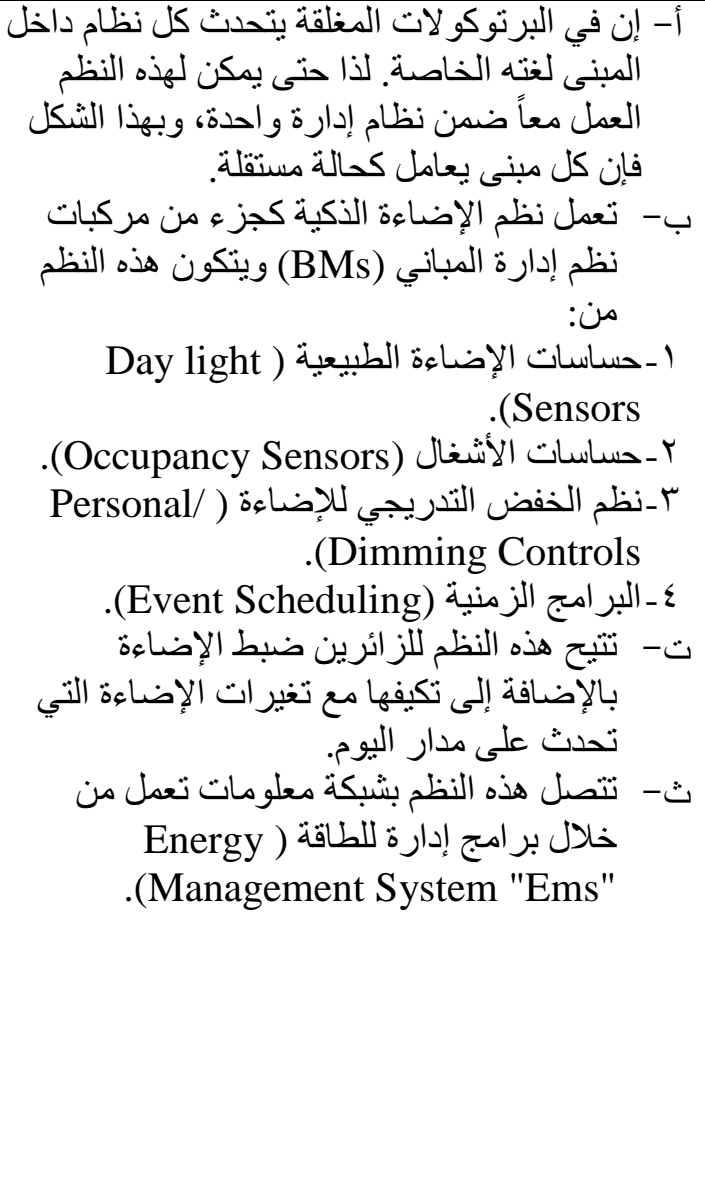 \\
\hline
\end{tabular}


هـ ـ مكونات نظام الإضاءة الذكية في غرف الفنادق:

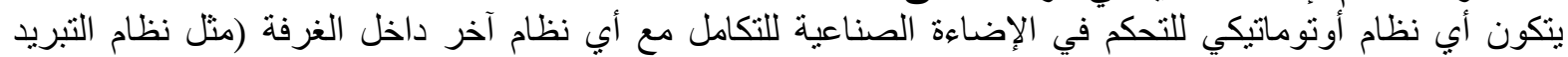
والتهوية "HVAC" - الإضـاءة الطبيعية Occupancy Natural Lighting) حيث يسمى "نظام الإضاءة الصناعية الطية

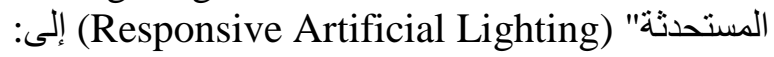

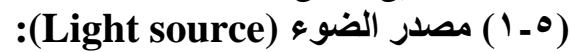
يتم اختبار الآن في غرف الفوء (الفنادق مصابيح أللإ لما فيها نوفير للطاقة سو اء كانت مصابيح - شر ائط.

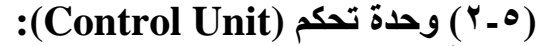
هى الأداة التي تستخدم في الجهد الداخل لمصدر الضوء. وهى نوعان إما أنظمة الفتح و الغلق (Switching) أو أنظمة

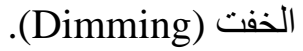

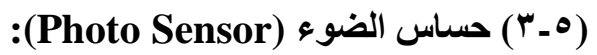

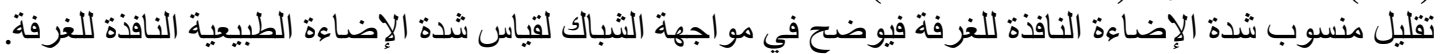
(0) (-) ) حساس الأشغال (Occupancy Sensors):

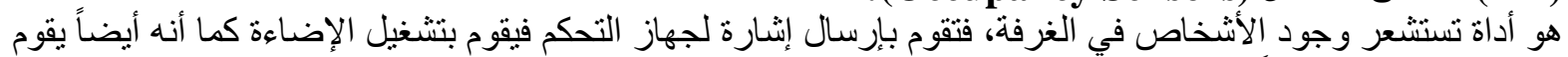

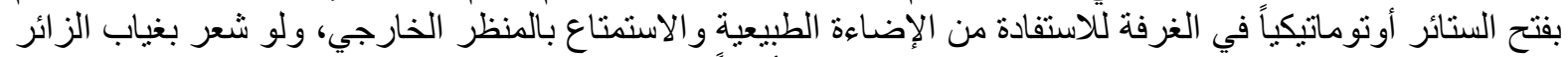

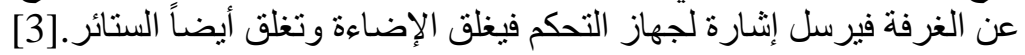

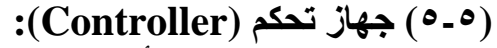
يعتبر "عقل نظام التحكم الأوتوماتيكي"، فهو المسئول عن تحديد التغير اللازم في منسوب شدة الإضـاءة فيقوم بغلق أو فتح

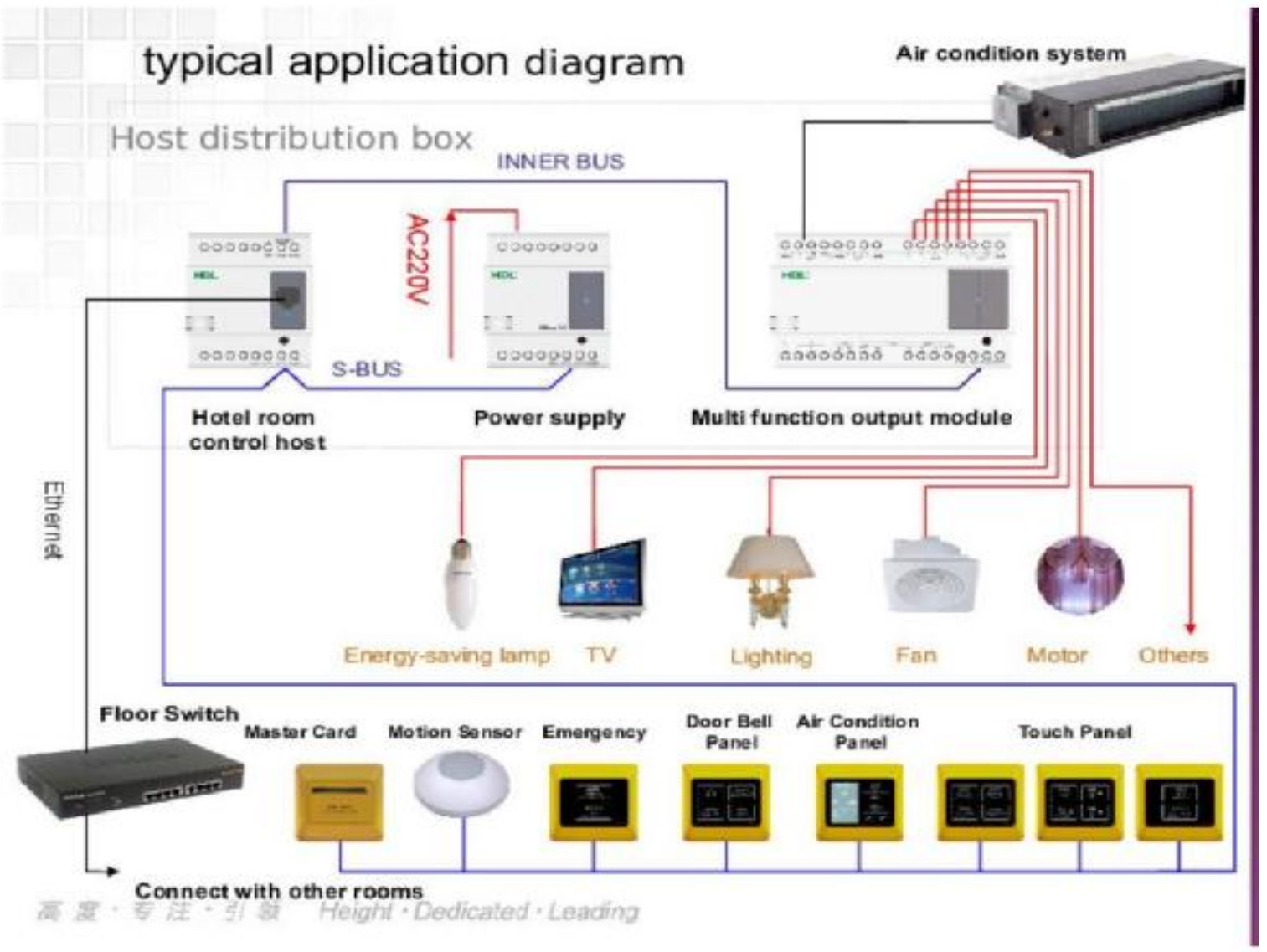

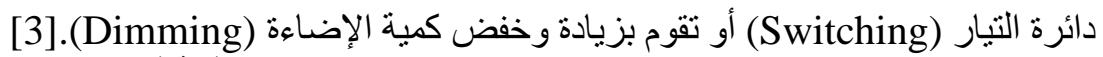

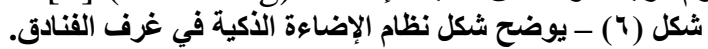

(www.nuuon.com/hotels/typical application diagram) 
צ · ت تحسين كفاعة الطاقة باستخدام الانظمة الذكية في الفنادق:

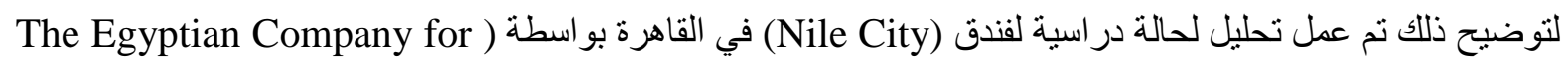

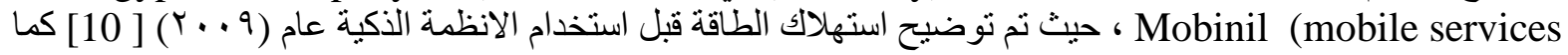
هو موضح في الجدول ( ( ) و الثكل (V).

\begin{tabular}{|c|c|c|c|c|c|c|}
\hline $\begin{array}{c}\text { Total } \\
\text { Consumption } \\
\text { in Kw.hr }\end{array}$ & $\begin{array}{c}\text { Average } \\
\text { Consumptio } \\
\mathbf{n} \text { in Kw.hr }\end{array}$ & $\begin{array}{c}\text { Average } \\
\text { Kw.hr/ } \\
\mathbf{m} 2\end{array}$ & $\begin{array}{c}\text { Max } \\
\text { Consumptio } \\
\mathbf{n} \text { in Kw.hr }\end{array}$ & $\begin{array}{c}\text { Min } \\
\text { Consumpti } \\
\text { on in } \\
\text { Kw.hr }\end{array}$ & $\begin{array}{c}\text { Max } \\
\text { Consumption } \\
\text { deviation } \\
\text { from average }\end{array}$ & $\begin{array}{c}\text { Min } \\
\text { Consumption } \\
\text { deviation } \\
\text { average }\end{array}$ \\
\hline $2,796,419$ & 233,035 & 10.44 & 267,961 & 210,259 & $14.99 \%$ & $-9.77 \%$ \\
\hline
\end{tabular}

The Egyptian Company for mobile services(Mobinil) : (المصدر)

Corporate presentation - March 1,1008 Nile City ,unrestricted).

\section{Kw.hr 2009}

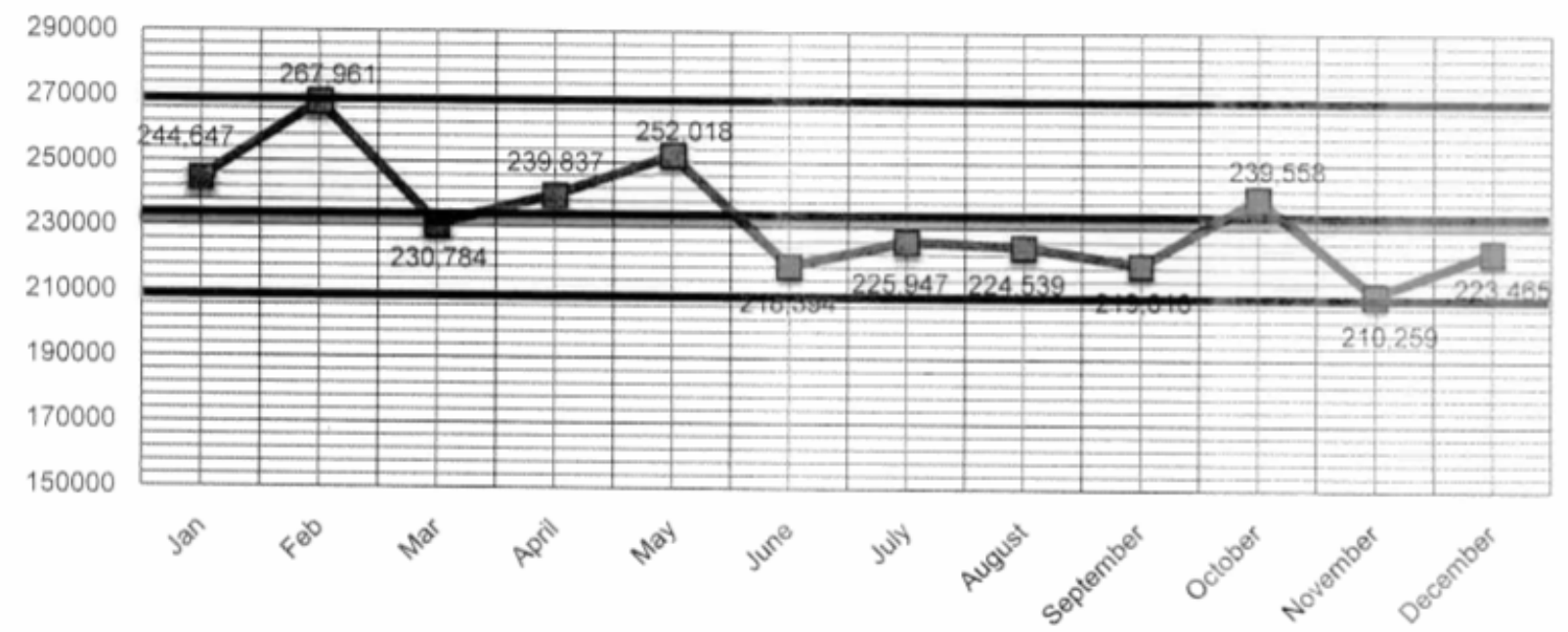

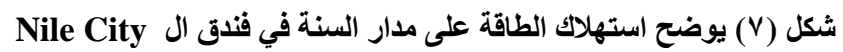

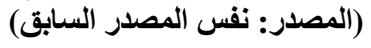

اما بعد استخدام الانظمة الذكية ABB Bsu - IB - (KNX) في عام • 1 . ب فقد قل استهلاك الطاقة وز ادت كفاءة

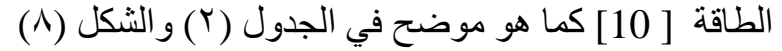

جدول (ץ) يوضح الفرق في كفاءة الطاقة بعد استخدام الانظمة الذكية

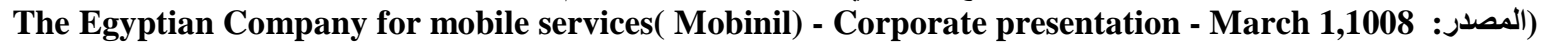

Nile City ,unrestricted).

\begin{tabular}{|c|c|c|c|c|c|c|c|}
\hline Building & $\begin{array}{c}\text { Total } \\
\text { Consump } \\
\text { tion in } \\
\text { Kw.hr }\end{array}$ & $\begin{array}{c}\text { Average } \\
\text { Consumpti } \\
\text { on in } \\
\text { Kw.hr }\end{array}$ & $\begin{array}{c}\text { Max } \\
\text { Consumption } \\
\text { in Kw.hr }\end{array}$ & $\begin{array}{c}\text { Min } \\
\text { Consumption } \\
\text { in Kw.hr }\end{array}$ & $\begin{array}{c}\text { Average } \\
\text { Kw.hr/m } \\
\mathbf{2}\end{array}$ & $\begin{array}{c}\text { Max } \\
\text { consumption } \\
\text { deviation from } \\
\text { foverage }\end{array}$ & $\begin{array}{c}\text { Minsumption } \\
\text { deviation } \\
\text { from average }\end{array}$ \\
\hline NC 2010 & $2,668,290$ & 222,358 & 253,573 & 195,759 & 9.96 & $14.04 \%$ & $-11.96 \%$ \\
\hline NC 2009 & $2,796,419$ & 233,035 & 267,961 & 210259 & 10.44 & $14.99 \%$ & $-9.77 \%$ \\
\hline
\end{tabular}


Kw.hr 2009 vs 2010

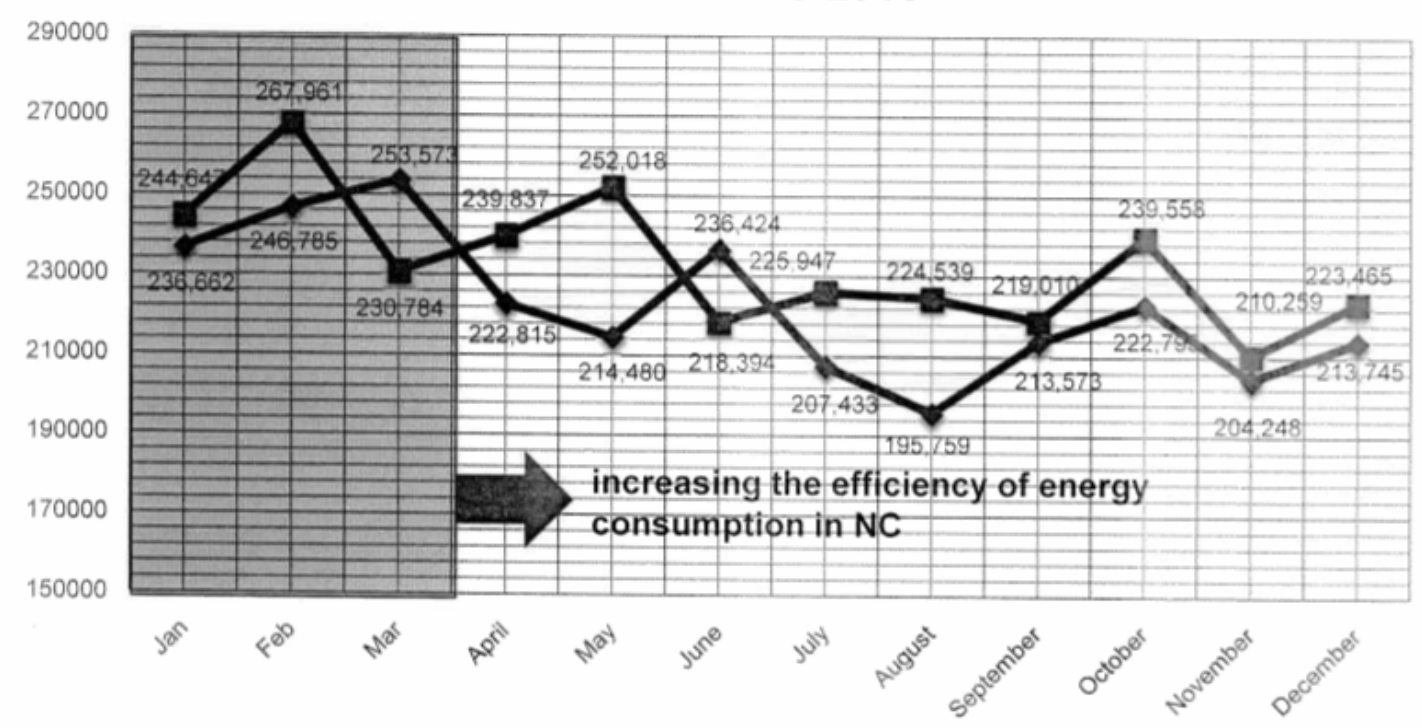

شكل (^) يوضح الزيادة في كفاءة الطاقة بعد استخدام الانظمة الأكية

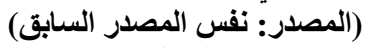

مما سبق نستنتج ان استخدام الانظمة الذكية يقلل من استهلاك الطاقة ويزيد من كفاءة الطاقة. • V

لتحقيق جودة البيئة الضوئية كجزء من تحقيق جودة البيئة الداخلية في غرف الفنادق يتم ذللك عن طريق:

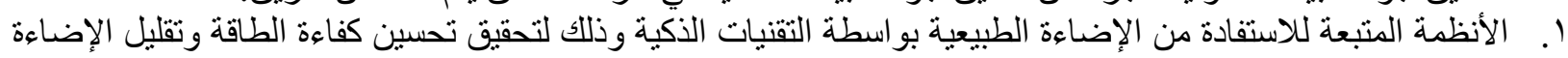

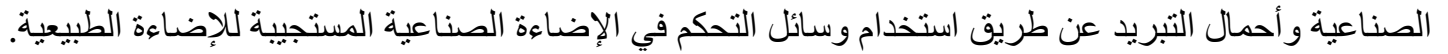

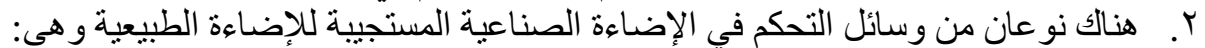

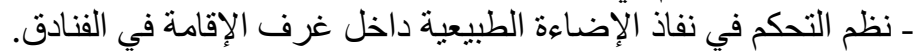

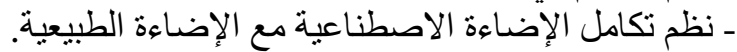

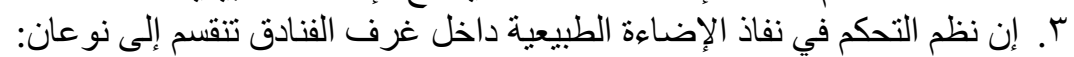

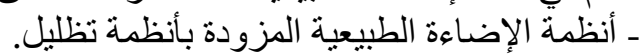

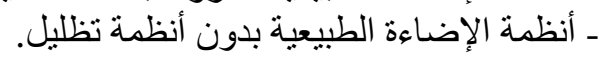

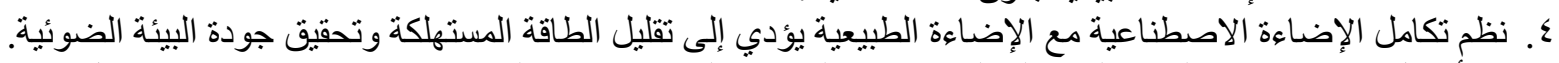

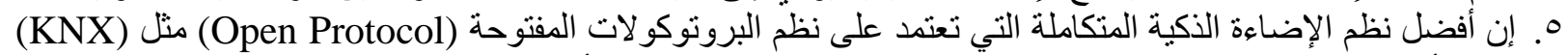

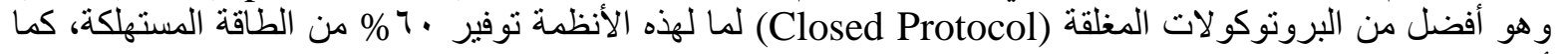
أنها مرنة ونتيح مجمو عة كبيرة من النظم و المعدات من خلال (Standard Software Interface).

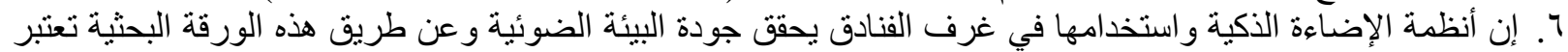

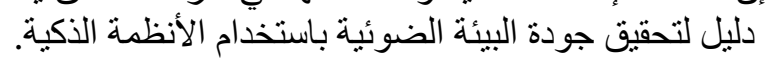
ا. . يوصى باستخدام الأنظمة الذكية في غرف الفنادق سواء كانت أنظمة الأمن والأمان (أنظمة الوقاية من الحريق ـ

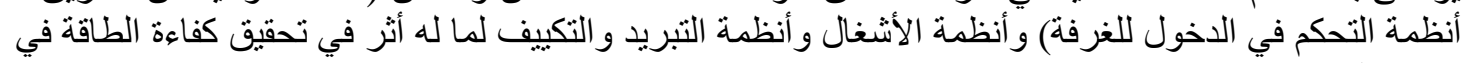

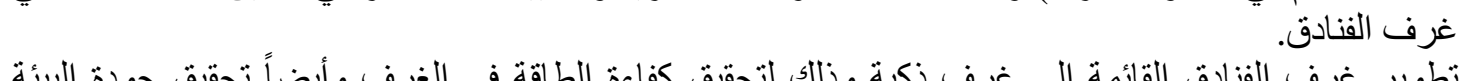
تطوير غرف الفنادق القائمة إلى غرف ذكية وذللك لتحقيق كفاءة الطاقة في الغرف وأيضاً تحقيق جودة البيئة الن الدلية.

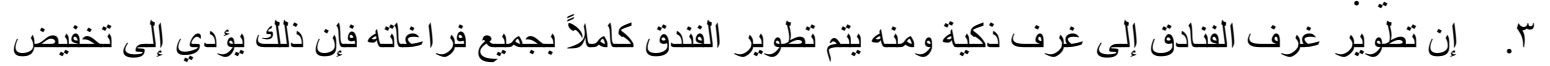

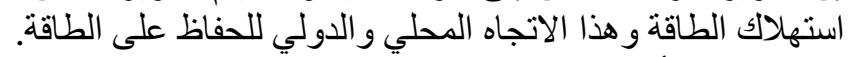

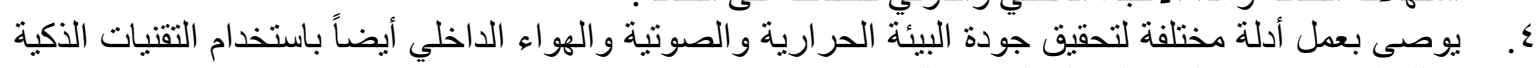

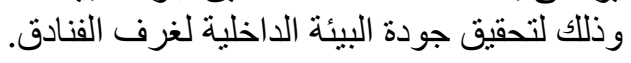




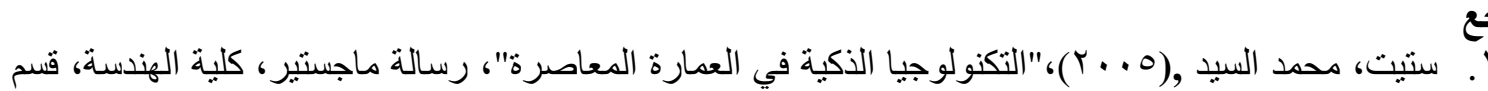

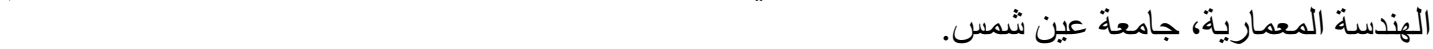

r. فجال، أحمد عاطف الدسوقي,(2010)، "العلاقة التكاملية بين مصادر الطاقة الطبيعية والتوافق البيئي في

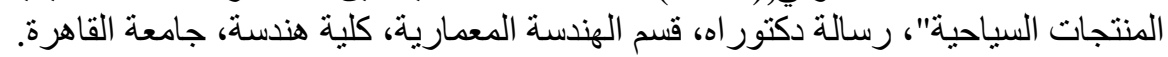

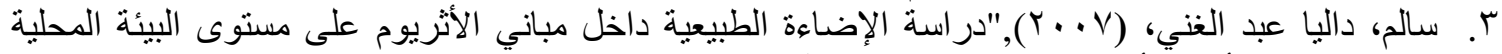
للوصول إلى الأداء الأمثل باستخدام الحاسب الآلي"، رسالة دكتور اه، قسم الهندسة المعمارية، كلية الهندسة،

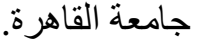

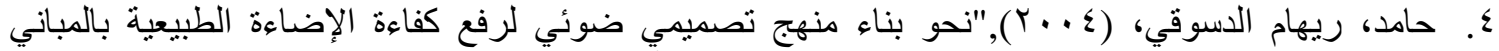

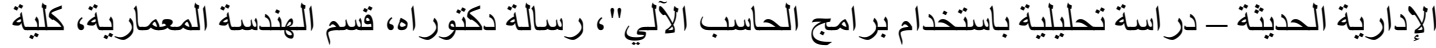

5. Richard Watkins,(2010),"Daylight in Building", Computer science..

6. www.desmena.com, (2018),W Hotel, Dubai by Ateliers Jean Nouvel.

7. www.1000Bulb.com,(2018), Hotel guest room lighting,.

8. www.bowinlock,com, (2017).

9. www.nuuon.com,(2019), typical application diagram .

10. The Egyptian Company for mobile services( Mobinil), - (March 1,2008), Corporate presentation , Nile City ,unrestricted, Electrical Analysis report for ABB KNX saving in Nile City in 2010 DOC. 